\title{
Supporting Graph-Based Real-Time Applications in Distributed Systems*
}

\author{
Cong Liu and James H. Anderson \\ Department of Computer Science, University of North Carolina at Chapel Hill
}

\begin{abstract}
The processing graph method (PGM) is a widely used framework for modeling applications with producer/consumer precedence constraints. PGM was originally developed by the U.S. Navy to model signal-processing applications where data communications exist among connected tasks. Prior work has shown how to schedule PGMspecified systems on uniprocessors and globally-scheduled multiprocessors. In this paper, this work is extended to enable such systems to be supported in a distributed collection of multicore machines. In such a context, pure global and partitioned scheduling approaches are problematic. Moreover, data communication costs must be considered. In this paper, a clustered scheduling algorithm is proposed for soft real-time PGM-specified distributed task systems for which bounded deadline tardiness is acceptable. This algorithm is effective in reducing data communication costs with little utilization loss. This is shown both analytically and via experiments conducted to compare it with an optimal integer linear programming solution.
\end{abstract}

\section{Introduction}

In work on real-time scheduling in distributed systems, task models where no inter-task precedence constraints exist, such as the periodic and the sporadic task models, have received much attention. However, in many real-time systems, applications are developed using processing graphs [7, 10], where vertices represent sequential code segments and edges represent precedence constraints. For example, multimedia applications and signal-processing algorithms are often specified using directed acyclic graphs (DAGs) $[9,10]$. With the growing prevalence of multicore platforms, it is inevitable that such DAG-based real-time applications will be deployed in distributed systems where multicore machines are used as per-node computers. One emerging example application where such a deployment is expected is fractionated satellite systems [8]. Such a system consists of a number of wirelessly-connected small satellites, each

\footnotetext{
*Work supported by NSF grants CNS 0834270, CNS 0834132, and CNS 1016954; ARO grant W911NF-09-1-0535; AFOSR grant FA9550-09-10549; and AFRL grant FA8750-11-1-0033.
}

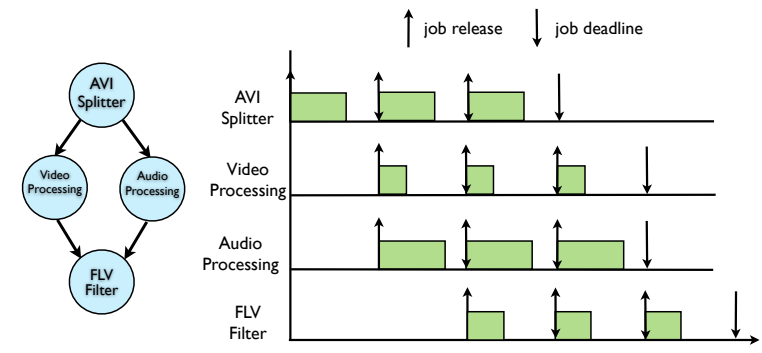

Figure 1: Example DAG.

of which may be controlled by a multicore machine. The overall collection of such machines is expected to support DAG-based real-time workloads such as radar and signalprocessing subsystems. To support such workloads, efficient scheduling algorithms are needed. Motivated by applications such as this, this paper is directed at supporting real-time DAG-based applications in distributed systems.

We view a distributed system as a collection of clusters of processors, where all processors in a cluster are locally connected (i.e., on a multicore machine). A DAG-based task system can be deployed in such a setting by $(i)$ assigning tasks to clusters, and (ii) determining how to schedule the tasks in each cluster. In addressing $(i)$, overheads due to data communications among connected tasks must be considered since tasks within the same DAG may be assigned to different clusters. In addressing (ii), any employed scheduling algorithm should seek to minimize utilization loss.

Within a cluster, precedence constraints can easily be supported by viewing all deadlines as hard and executing tasks sporadically (or periodically), with job releases adjusted so that successive tasks execute in sequence. Fig. 1 shows an example multimedia application, which transforms an input AVI video file into a FLV video file (AVI and FLV are two types of multimedia container formats). This application is represented by a DAG with four sporadic tasks. (DAG-based systems are formerly defined in Sec. 2.) Fig. 1 shows a global-earliest-deadline-first (GEDF) schedule for this application on a two-processor cluster. (It suffices to know here that the $k^{t h}$ job of the AVI splitter task, the video processing task [or the audio processing task], and the FLV filter task, respectively, must execute in sequence.) As seen in this example, the timing guarantees provided by the sporadic model ensure that any DAG executes correctly as long as no deadlines are missed. 
Unfortunately, if all deadlines of tasks assigned to the same cluster must be viewed as hard, then significant processing capacity must be sacrificed, due to either inherent schedulability-related utilization loss-which is unavoidable under most scheduling schemes [6]—or high runtime overheads-which typically arise in optimal schemes that avoid schedulability-related loss [4]. In systems where less stringent notions of real-time correctness suffice, such utilization loss can be avoided by viewing deadlines as soft. In this paper, such systems are our focus; the notion of soft real-time correctness we consider is that deadline tardiness is bounded.

To the best of our knowledge, in all prior work on supporting DAG-based applications in systems with multiple processors (multiprocessors or distributed systems), either global or partitioned scheduling has been assumed. Under global scheduling, tasks are scheduled from a single run queue and may migrate across processors; in contrast, under partitioned schemes, tasks are statically bound to processors and per-processor schedulers are used. Partitioned approaches are susceptible to bin-packing-related schedulability limitations, which global approaches can avoid. Indeed, if bounded deadline tardiness is the timing constraint of interest, then global approaches can often be applied on multiprocessor platforms with no loss of processing capacity [13]. However, the virtues of global scheduling come at the expense of higher runtime overheads. In work on ordinary sporadic (not DAG-based) task systems, clustered scheduling, which combines the advantages of both global and partitioned scheduling, has been suggested as a compromise $[3,5]$. Under clustered scheduling, tasks are first partitioned onto clusters of cores, and intra-cluster scheduling is global.

In distributed systems, clustered scheduling algorithms are a natural choice, given the physical layout of such a system. Thus, such algorithms are our focus here. Our specific objective is to develop clustered scheduling techniques and analysis that can be applied to support DAGs, assuming that bounded deadline tardiness is the timing guarantee that must be ensured. Our primary motivation is to develop such techniques for use in distributed systems, where different clusters are physically separated; however, our results are also applicable in settings where clusters are tightly coupled (e.g., each cluster could be a socket in a multi-socket system). Our results can be applied to systems with rather sophisticated precedence constraints. To illustrate this, we consider a particularly expressive DAG-based formalism, the processing graph method (PGM) [12]. PGM was first developed by the U.S. Navy to model signal-processing applications where producer/consumer relationships exist among tasks. In a distributed system, it may be necessary to transfer data from a producer in one cluster to a consumer in another through an inter-cluster network, which could cause a significant amount of data communication overhead. Thus, any proposed scheduling algorithm should seek to minimize such inter-cluster data communication.

Related work. To our knowledge, DAGs have not been considered before in the context of clustered real-time scheduling algorithms, except for the special cases of partitioned and global approaches. ${ }^{1}$ An overview of work on scheduling DAGs in a distributed system under partitioned approaches (which we omit here due to space constraints) can be found in [14]. The issue of scheduling PGM graphs on a uniprocessor was extensively considered by Goddard in his dissertation [10]. Goddard presented techniques for mapping PGM nodes to tasks in the rate-based-execution (RBE) task model [11], as well as conditions for verifying the schedulability of the resulting task set under a rate-based, earliest-deadline-first (EDF) scheduler.

In recent work [14], we extended Goddard's work and showed that a variety of global scheduling algorithms can ensure bounded deadline tardiness in general DAG-based systems with no utilization loss on multiprocessors, including algorithms that are less costly to implement than optimal algorithms.

Contributions. In this paper, we show that sophisticated notions of acyclic precedence constraints can be efficiently supported under clustered scheduling in a distributed system, provided bounded deadline tardiness is acceptable. The types of precedence constraints we consider are those allowed by PGM. We propose a clustered scheduling algorithm called CDAG that first partitions PGM graphs onto clusters, and then uses global scheduling approaches within each cluster. We present analysis that gives conditions under which each task's maximum tardiness is bounded. Any clustered approach is susceptible to some bin-packing-related utilization loss; however, the conditions derived for CDAG show that for it, such loss is small. To assess the effectiveness of CDAG in reducing inter-cluster data communications, we compare it with an optimal integer linear programming (ILP) solution that minimizes inter-cluster data communications when partitioning PGM graphs onto clusters. We assume that tasks are specified using a rate-based task model that generalizes the periodic and sporadic task models.

Organization. The rest of this paper is organized as follows. Sec. 2 describes our system model. In Sec. 3, the ILP formulation of the problem and the polynomial-time CDAG algorithm and its analysis are presented. Sec. 4 evaluates the proposed algorithm via an experimental study. Sec. 5 concludes.

\footnotetext{
${ }^{1}$ The problem of non-real-time DAG scheduling in parallel and distributed systems has been extensively studied. An overview on such work can be found in [15].
} 


\section{Preliminaries}

In this section, we present an overview of PGM and describe the assumed system architecture. For a complete description of the PGM, please see [12].

PGM specifications. An acyclic PGM graph system [12] consists of a set of acyclic PGM graphs (DAGs), each with a distinct source node. Each PGM graph contains a number of nodes connected by edges. A node can have outgoing or incoming edges. A source node, however, can only have outgoing edges. Each directed edge in a PGM graph is a typed first-in-first-out queue, and all nodes in a graph are assumed to be reachable from the graph's source node. A producing node transports a certain number of data units ${ }^{2}$ to a consuming node, as indicated by the data type of the corresponding queue. Data is appended to the tail of the queue by the producing node and read from the head by the consuming node. A queue is specified by three attributes: a produce amount, a threshold, and a consume amount. The produce amount specifies the number of data units appended to the queue when the producing node completes execution. The threshold amount specifies the minimum number of data units required to be present in the queue in order for the consuming node to process any received data. The consume amount is the number of data units dequeued when processing data. We assume that the queue that stores data is associated with the consuming node. That is, data is stored in memory local to the corresponding consuming node. ${ }^{3}$ The only restriction on queue attributes is that they must be non-negative integral values and the consume amount must be at most the threshold. In the PGM framework, a node is eligible for execution when the number of data units on each of its input queues is over that queue's threshold. Overlapping executions of the same node are disallowed. For any queue connecting nodes $T_{l}^{j}$ and $T_{l}^{k}$ in a PGM graph $T_{l}$, we let $\rho_{l}^{j k}$ denote its produce amount, $\theta_{l}^{j k}$ denote its threshold, and $\sigma_{l}^{j k}$ denote the consume amount. If there is an edge from task $T_{l}^{k}$ to task $T_{l}^{h}$ in graph $T_{l}$, then $T_{l}^{k}$ is called a predecessor task of $T_{l}^{h}$. We let $\operatorname{pred}\left(T_{l}^{h}\right)$ denote the set of all predecessor tasks of $T_{l}^{h}$. We define the depth of a task within a graph to be the number of edges on the longest path between this task and the source task of the corresponding graph.

Example. Fig. 2(a) shows an example PGM graph system consisting of two graphs $T_{1}$ and $T_{2}$ where $T_{1}$ contains four nodes with four edges and $T_{2}$ contains two nodes with one edge. We will use this example to illustrate other concepts throughout the paper.

In the PGM framework, it is often assumed that each source node executes according to a rate-based pattern (see

\footnotetext{
${ }^{2} \mathrm{We}$ assume that data units are defined so that the number of such units produced reflects the total size of the data produced.

${ }^{3}$ It is more reasonable to associate a queue with the corresponding consuming node than with the producer node because this enables the consumer to locally detect when the queue is over threshold and react accordingly.
}

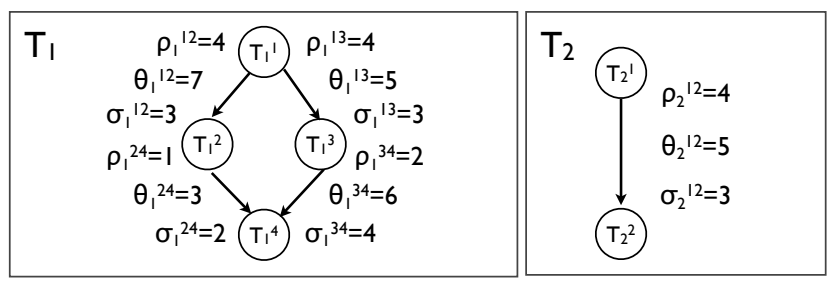

(a) Example PGM graph system

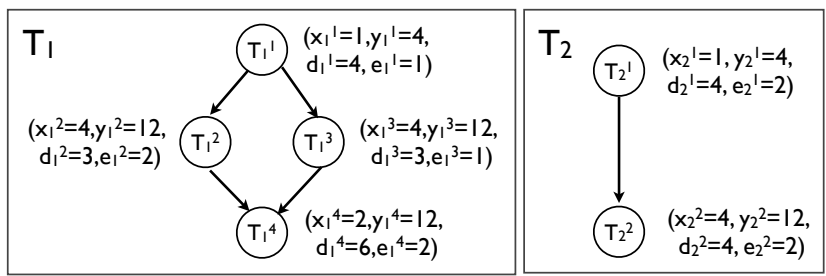

(b) Rate-based counterpart of (a)

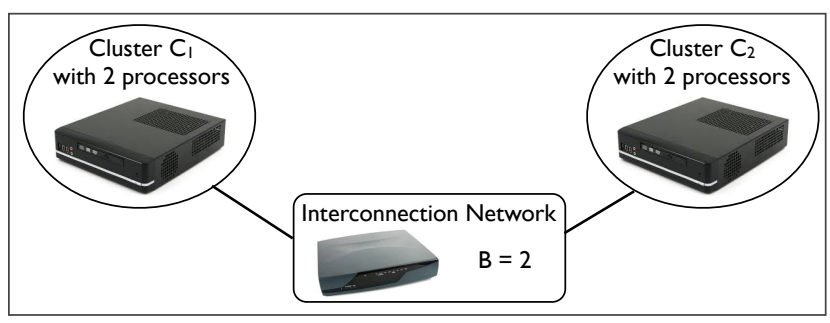

(c) An example distributed system

Figure 2: Example system used throughout the paper.

below). Note that, even if all source nodes execute according to a periodic/sporadic pattern, non-source nodes may still execute following a rate-based pattern [10,14]. The execution rate of a node within a PGM graph can be calculated based upon the attributes of the node's incoming edges using techniques presented in $[10,14]$.

According to the rate-based task model [11,14], each task $T_{l}^{h}$ within a PGM graph $T_{l}$ is specified by four parameters: $\left(x_{l}^{h}, y_{l}^{h}, d_{l}^{h}, e_{l}^{h}\right)$. The pair $\left(x_{l}^{h}, y_{l}^{h}\right)$ represents the maximum execution rate of $T_{l}^{h}: x_{l}^{h}$ is the maximum number of invocations of the task in any interval $\left[j \cdot y_{l}^{h},(j+1) \cdot y_{l}^{h}\right)(j \geq 0)$ of length $y_{l}^{h}$; such an invocation is called a job of $T_{l}^{h} . x_{l}^{h}$ and $y_{l}^{h}$ are assumed to be non-negative integers. Additionally, $d_{l}^{h}$ is the task's relative deadline, and $e_{l}^{h}$ is its worst-case execution time. $d_{l}^{h}$ is defined to be

$$
d_{l}^{h}=y_{l}^{h} / x_{l}^{h} .
$$

The $j^{\text {th }}$ job of $T_{l}^{h}$ is denoted $T_{l, j}^{h}$. We denote its release time (when $T_{l}^{h}$ is invoked) as $r_{l, j}^{h}$ and its (absolute) deadline as

$$
d_{l, j}^{h}=r_{l, j}^{h}+d_{l}^{h} .
$$

The utilization of $T_{l}^{h}, u_{l}^{h}$, is defined to be $e_{l}^{h} \cdot \frac{x_{l}^{h}}{y_{l}^{h}}$. The utilization of a PGM graph $T_{l}$ is defined to be $u_{l}=$ $\sum_{T_{l}^{h} \in T_{l}} u_{l}^{h}$. The widely-studied sporadic task model is a special case of the rate-based task model. In the sporadic 


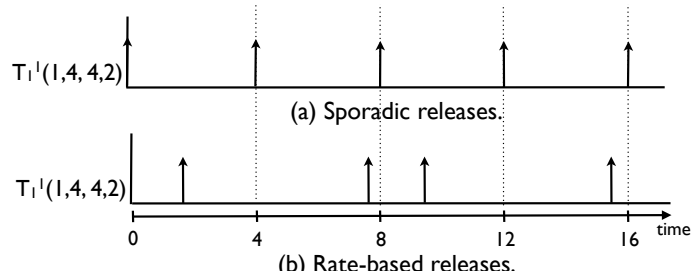

Figure 3: Sporadic and rate-based releases.

task model, a task is released no sooner than every $p$ time units, where $p$ is the task's period. In the rate-based task model, the notion of a "rate" is much more general.

Example. Fig. 2(b) shows the rate-based counterpart of the PGM graphs in Fig. 2(a). Fig. 3 shows job release times for task $T_{1}^{1}(1,4,4,1)$ of graph $T_{1}$. In inset (a), jobs are released sporadically, once every four time units in this case. Inset (b) shows a possible job-release pattern that is not sporadic. As seen, the second job is released at time 7.5 while the third job is released at time 9.5. The separation time between these jobs is less than that seen in the sporadic schedule.

A job $T_{l, j}^{h}$ may be restricted from beginning execution until certain jobs of tasks in $\operatorname{pred}\left(T_{l}^{h}\right)$ have completed (i.e., $T_{l, j}^{h}$ cannot start execution until receiving the required data from these jobs). We denote the set of such predecessor jobs as $\operatorname{pred}\left(T_{l, j}^{h}\right)$. $\operatorname{pred}\left(T_{l, j}^{h}\right)$ can be defined precisely by examing the execution rates of all tasks on any path from the source node of $T_{l}$ to $T_{l}^{h}$ [14]. We require that for any job $T_{l, j}^{h}$ and one of its predecessor jobs, $T_{i, v}^{w}$,

$$
r_{l, j}^{h} \geq r_{i, v}^{w}
$$

holds.

If a job $T_{l, j}^{h}$ completes at time $t$, then its tardiness is defined as $\max \left(0, t-d_{l, j}^{h}\right)$ and its response time is defined as $\max \left(0, t-r_{l, j}^{h}\right)$. A PGM graph's tardiness is the maximum of the tardiness of any job of any of its tasks. Note that, when a job of a task misses its deadline, the release time of the next job of that task is not altered. However, jobs of the same task still cannot execute in parallel.

System architecture. We consider the problem of scheduling a set of $n$ acyclic PGM graphs on $\varphi$ clusters $C_{1}, C_{2}, \ldots, C_{\varphi}$. Each cluster $C_{i}$ contains $\lambda_{i}$ processors. Clusters are connected by a network. Let $B$ denote the minimum number of data units that can be transferred between any two clusters per time unit. Similarly, let $b$ denote the minimum number of data units that can be transferred between any two processors within the same cluster per time unit. If the system is a distributed collection of multicore machines, then $B$ is impacted by the speed and bandwidth of the communication network and $b$ is impacted by the speed of the data transfer bus on a multicore chip. In this case, $b \gg B$, as local on-chip data communication is generally much faster than communication across a network.
Example. An example distributed system containing two clusters each with two processors interconnected by a network is shown in Fig. 2(c).

\section{Algorithm CDAG}

In this section, we propose Algorithm CDAG, a clusteredscheduling algorithm that ensures bounded tardiness for DAG-based systems on distributed clusters. Since intercluster data communication can be expensive, CDAG is designed to reduce such communication.

CDAG consists of two phases: an assignment phase and an execution phase. The assignment phase executes offline and assigns each PGM graph to one or more clusters. In the execution phase, PGM graphs are first transformed to ordinary sporadic tasks (where no precedence constraints arise), and then scheduled under a proposed clustered scheduling algorithm.

\subsection{Assignment Phase}

The assignment phase assigns acyclic PGM graphs (or DAGs for short) to clusters in a way such that the intercluster data communication cost is reduced. Note that the total utilization of the DAGs (or portions of DAGs) assigned to a cluster must not exceed the total capacity of that cluster. CDAG contains an assignment algorithm that is designed to partition DAGs onto clusters such that both the inter-cluster data communication cost and any bin-packing-related utilization loss are minimized.

To provide a better understanding of the problem of partitioning DAGs onto clusters with minimum inter-cluster data communication cost, we first formulate it as an ILP, which provides an optimal solution. (Note that, due to potential utilization loss arising from partitioning DAGs, the ILP approach may not find a feasible assignment. However, if the ILP approach cannot find a feasible solution, then the given task set cannot be assigned to clusters under any partitioning algorithm.)

Definition 1. For any edge $e_{i}^{j k}$ of DAG $T_{i}$, its edge data weight, $w_{i}^{j k}$, is defined to be $\rho_{i}^{j k} \cdot \frac{x_{i}^{j}}{y_{i}^{j}}$. A larger edge data weight indicates a larger data communication cost between tasks connected by the corresponding edge. For any edge $e_{i}^{j k}$ of DAG $T_{i}$, its communication cost $\varpi_{i}^{j k}$ is defined to be $w_{i}^{j k}$ if the corresponding connected nodes $T_{i}^{j}$ and $T_{i}^{k}$ are assigned to different clusters, and 0 otherwise.

Note that the above definition does not consider data consuming rates. This is because data is stored in memory local to the consuming node, as discussed in Sec. 2. Thus, only produced data needs to be transferred.

Example. For task $T_{1}^{1}$ of DAG $T_{1}$ in Fig. 2, we can use previous techniques $[10,14]$ to calculate its execution rates, 
which are $x_{1}^{1}=1$ and $y_{1}^{1}=4$. Thus, for edge $e_{1}^{12}$ of $T_{1}$, its edge data weight $w_{1}^{12}=\rho_{1}^{12} \cdot \frac{x_{1}^{1}}{y_{1}^{1}}=4 \cdot \frac{1}{4}=1$. Intuitively, node $T_{1}^{1}$ produces one data unit on average on edge $e_{1}^{12}$ per time unit, given its execution rate $\left(x_{1}^{1}, y_{1}^{1}\right)=(1,4)$.

Definition 2. The total communication cost of any given DAG-based system $\tau$, denoted $\varpi_{\text {sum }}$, is given by $\sum_{T_{i} \in \tau} \sum_{e_{i}^{j k} \in T_{i}} \varpi_{i}^{j k}$.

ILP formulation. We are given a set $\tau$ of tasks (each task corresponds to a node in a DAG) and a set $\xi$ of clusters. To reduce clutter in the ILP formulation, we denote tasks more simply as $T_{1}, T_{2}, \ldots$, and let $w_{i, j}$ be the data weight of the edge connecting tasks $T_{i}$ and $T_{j}\left(w_{i, j}=0\right.$ if $i$ and $j$ are not connected). (This simplified notation is used only for the ILP formulation.)

For all $T_{i} \in \tau$, let $x_{i, k}$ be a binary decision variable that equals 1 when task $T_{i}$ is assigned to cluster $C_{k}$, and 0 otherwise. For all $\left(T_{i}, T_{j}\right) \in \tau \times \tau$, let $y_{i, j}$ be a binary decision variable that equals 1 if tasks $T_{i}$ and $T_{j}$ are assigned to the same cluster, and 0 otherwise.

Our goal is to minimize the total communication cost. An ILP formulation of this optimization problem is then:

Minimize

$$
\sum_{i \in \tau} \sum_{j \in \tau} w_{i, j} \cdot\left(1-y_{i, j}\right)
$$

subject to the constraints below. Note that by Defs. 1 and 2, (4) represents the total communication cost.

- Each task must be assigned to one cluster:

$$
\sum_{C_{k} \in \xi} x_{i, k}=1, \forall T_{i} \in \tau .
$$

- The total utilization of all tasks assigned to a cluster must not exceed the total capacity of that cluster:

$$
\sum_{T_{i} \in \tau} x_{i, k} \cdot u_{i} \leq \lambda_{k}, \forall C_{k} \in \xi .
$$

- $y_{i, j}$ should be 1 when two tasks are assigned to the same cluster, and 0 otherwise:

$$
\begin{gathered}
y_{i, j} \leq x_{i, k}-x_{j, k}+1, \forall\left(T_{i}, T_{j}\right) \in \tau \times \tau, \forall C_{k} \in \xi, \\
y_{i, j} \leq-x_{i, k}+x_{j, k}+1, \forall\left(T_{i}, T_{j}\right) \in \tau \times \tau, \forall C_{k} \in \xi .
\end{gathered}
$$

By solving the ILP above, we obtain an optimal assignment that gives the minimum total communication cost as long as there exists a feasible assignment.

Example. Consider assigning DAGs $T_{1}$ and $T_{2}$ in Fig. 2 to two clusters. $T_{1}$ has a utilization of $1 / 4+2 / 3+1 / 3+1 / 3=$ $19 / 12$ and $T_{2}$ has a utilization of $1 / 2+2 / 3=7 / 6$. By formulating this assignment problem as an ILP according to the above approach, an optimal solution is to assign all tasks of $T_{1}$ to the first cluster and all tasks of $T_{2}$ to the second cluster, which leads to $\varpi_{\text {sum }}=0$.

\section{ASSIGN}

$u\left(C_{i}\right)$ : CAPACITY OF CLUSTER $C_{i}$, INITIALly $u\left(C_{i}\right)=\lambda_{i}$ $\xi$ : A LiST OF CLUSTERS $\left\{C_{1}, C_{2}, \ldots, C_{\varphi}\right\}$

$\zeta$ : A LIST OF DAGs $\left\{T_{1}, T_{2}, \ldots, T_{n}\right\}$

\section{Phase 1:}

1 Order DAGs in $\zeta$ by largest average data weight first

2 Order clusters in $\xi$ by smallest capacity first

3 for each DAG $T_{i}$ in $\zeta$ in order

4 for each cluster $C_{j}$ in $\xi$ in order

$5 \quad$ if $u_{i} \leq u\left(C_{j}\right)$

$6 \quad$ Assign all tasks of $T_{i}$ to $C_{j}$

$7 \quad$ Remove $T_{i}$ from $\zeta ; u\left(C_{j}\right):=u\left(C_{j}\right)-u_{i}$

Phase 2:

8 for each DAG $T_{i}$ in $\zeta$ in order

9 Order tasks within $T_{i}$ by smallest depth first, then order tasks within $T_{i}$ and at the same depth by largest task data weight first

10 Order clusters in $\xi$ by largest capacity first

11 for each task $T_{i}^{k}$ of DAG $T_{i}$ in $\zeta$ in order

12 for each cluster $C_{j}$ in $\xi$ in order

13 if $u_{i}^{k} \leq u\left(C_{j}\right)$ then

14

15

Figure 4: Psuedocode of the assignment algorithm.

A polynomial-time assignment algorithm. Although the ILP solution is optimal, it has exponential time complexity. We now propose a polynomial-time algorithm to assign DAGs to clusters. This algorithm tries to minimize the total communication cost, which is achieved by locally minimizing communication costs when assigning each DAG.

Definition 3. For any task $T_{i}^{j}$ of DAG $T_{i}$, its task data weight, $w_{i}^{j}$, is defined to be $\sum_{T_{i}^{j} \rightarrow T_{i}^{k}} w_{i}^{j k}$, where $T_{i}^{j} \rightarrow T_{i}^{k}$ denotes that $T_{i}^{j}$ has an outgoing edge to $T_{i}^{k}$.

Definition 4. For any DAG $T_{i}$, its average data weight, $w_{i}$, is defined to be $\frac{\sum_{e_{i}^{j k} \in T_{i}} w_{i}^{j k}}{E_{i}}$, where $E_{i}$ is the total number of edges in $T_{i}$. A DAG $T_{i}$ 's data weight is defined to be $\sum_{e_{i}^{j k} \in T_{i}} w_{i}^{j k}$.

Example. For DAG $T_{1}$ in Fig. 2, by Def. $1, w_{1}^{12}=w_{1}^{13}=$ $1, w_{1}^{24}=1 / 3$, and $w_{1}^{34}=2 / 3$. Thus, by Def. $4, T_{1}$ has an average data weight of $\frac{3}{4}$. By Def. 3, task $T_{1}^{1}$ of $T_{1}$ has a data weight of $w_{1}^{1}=w_{1}^{12}+w_{1}^{13}=2$. Intuitively, node $T_{1}^{1}$ produces two data units on average on its outgoing edges per time unit, given its execution rate $\left(x_{1}^{1}, y_{1}^{1}\right)=(1,4)$.

The proposed DAG assignment algorithm, denoted $A S$ $S I G N$, is shown in Fig. 4.

Algorithm description. Algorithm ASSIGN assigns DAGs to clusters in two phases. In the first phase (lines 1-7), it assigns DAGs in largest-average-data-weight-first order to 
clusters in smallest-capacity-first order, which gives a higher possibility for DAGs with larger average data weight to be fully assigned to a cluster. DAGs that cannot be assigned to clusters in the first phase are considered in the second phase (lines 8-15). For each unassigned DAG in order, its tasks are ordered by depth and then tasks at the same depth are ordered by data weight, which gives tasks with larger data weight a greater chance to be assigned to the same cluster as their predecessor tasks (lines 8-9). Then each task in order is assigned to clusters in largest-capacity-first order (lines 10-15). Task $T_{i}^{k}$ is assigned to cluster $C_{j}$ if $T_{i}^{k}$ can receive its full share of its utilization from $C_{j}$ (lines 13-14). If not, then $C_{j}$ is excluded from being considered for any of the later tasks, and the next cluster in order will be considered for scheduling $T_{i}^{k}$ (line 15).

Example. Consider the example DAG system in Fig. 2 to be partitioned under the proposed algorithm. By Defs. 1 and 2, $T_{1}$ has an average data weight of $3 / 4$ and $T_{2}$ has an average data weight of 1 . Thus, $T_{2}$ is ordered before $T_{1}$. Since $T_{2}$ has a utilization of $7 / 6$ and $T_{1}$ has a utilization of $19 / 12$, the tasks of $T_{2}$ are assigned to the first cluster, and the tasks of $T_{1}$ are assigned to the second cluster, which leads to $\varpi_{\text {sum }}=0$.

Time complexity. The time complexity of Phase 1 of $A S$ $S I G N$ depends on (i) the sorting process (lines 1-2), which is $O(n \cdot \log n+\varphi \cdot \log \varphi)$, and (ii) the two for loops (lines 3-4), which is $O(N \cdot \varphi)$, where $N$ is the number of tasks in the system (each task corresponds to a node in a DAG). Thus, Phase 1 has a time complexity of $O(n \cdot \log n+\varphi$. $\log \varphi+N \cdot \varphi)$. The time complexity of Phase 2 of $A S$ SIGN depends on (i) the sorting process (lines 9-10), which is $O(n \cdot \mu \cdot \log \mu+\varphi \cdot \log \varphi)$, where $\mu$ is the maximum number of tasks per-DAG, and (ii) the two for loops (lines 11-12), which is $O(N \cdot \varphi)$. Thus, Phase 2 has a time complexity of $O(n \cdot \mu \cdot \log \mu+\varphi \cdot \log \varphi+N \cdot \varphi)$. The overall time complexity of ASSIGN is thus $O(n \cdot \log n+\varphi \cdot \log \varphi+n \cdot \mu \cdot \log \mu+N \cdot \varphi)$.

Partitioning condition. The following theorem gives a condition for ASSIGN to successfully partition any given DAG-based task system onto clusters. For conciseness, let us denote tasks (each task corresponds to a node in a DAG) after ordering by $T_{1}, T_{2}, \ldots, T_{N}$ (note that tasks within DAGs that are fully assigned to clusters in the first phase are assumed to be ordered before all other tasks here). Let $u\left(T_{i}\right)$ denote the utilization of task $T_{i}$ under this notation. Before stating the theorem, we first prove the following lemma.

Lemma 1. Under Algorithm ASSIGN, if a task $T_{i}$ is the first task that cannot be assigned to any cluster, then $\sum_{k=1}^{i} u\left(T_{k}\right)>m-u_{\varphi-1}$, where $u_{\varphi-1}$ is the sum of $\varphi-1$ largest task utilizations.

Proof. Due to the fact that some task $T_{i}$ cannot be assigned to any cluster, the second phase of Algorithm ASSIGN is executed. In the second phase, if Algorithm ASSIGN fails to assign the $i^{\text {th }}$ task $T_{i}$ to any cluster, then the last cluster $C_{\varphi}$ does not have enough capacity to accommodate $T_{i}$. Moreover, for each previous cluster $C_{j}$, where $j \leq \varphi-1$, there exists a task, denoted $T^{j}$, that could not be assigned to $C_{j}$, and thus the next cluster in order was considered to accommodate $T^{j}$ and $C_{j}$ was removed from being considered again for any of the later tasks (line 15). That is, for each such cluster $C_{j}$, its remaining capacity is strictly less than the utilization of $T^{j}$ (for the last cluster, we know that $T^{\varphi}$ is $T_{i}$ ). Thus, for any cluster $C_{j}$, its allocated capacity is strictly greater than $\lambda_{j}-u\left(T^{j}\right)$. Since tasks $\left\{T_{1}, T_{2}, \ldots, T_{i-1}\right\}$ have been successfully assigned, the total utilization of these tasks is equal to the total allocated capacity of clusters, which is given by $\sum_{k=1}^{i-1} u\left(T_{k}\right)$. Hence, we have

$$
\begin{array}{ll} 
& \sum_{k=1}^{i-1} u\left(T_{k}\right)>\sum_{j=1}^{\varphi}\left(\lambda_{j}-u\left(T^{j}\right)\right) \\
\Leftrightarrow \quad & \left\{\text { adding } u\left(T_{i}\right) \text { on both sides }\right\} \\
& \sum_{k=1}^{i} u\left(T_{k}\right)>\sum_{j=1}^{\varphi}\left(\lambda_{j}-u\left(T^{j}\right)\right)+u\left(T_{i}\right) \\
\Leftrightarrow \quad & \left\{\text { because } \sum_{j=1}^{\varphi} \lambda_{j}=m \text { and } u\left(T^{\varphi}\right)=u\left(T_{i}\right)\right\} \\
& \sum_{k=1}^{i} u\left(T_{k}\right)>m-\sum_{j=1}^{\varphi-1} u\left(T^{j}\right) \\
\Rightarrow \quad\left\{\text { by the definition of } u_{\varphi-1}\right\} & \sum_{k=1}^{i} u\left(T_{k}\right)>m-u_{\varphi-1} .
\end{array}
$$

Theorem 1. Algorithm ASSIGN successfully partitions any DAG-based task system $\tau$ on $\varphi$ clusters for which $u_{\text {sum }} \leq$ $m-u_{\varphi-1}$.

Proof. Let us suppose that Algorithm ASSIGN fails to assign the $i^{t h}$ task $T_{i}$ to any cluster. Then by Lemma 1 , $\sum_{k=1}^{i} u\left(T_{k}\right)>m-u_{\varphi-1}$ holds. Therefore, we have

$$
\begin{gathered}
\sum_{k=1}^{i} u\left(T_{k}\right)>m-u_{\varphi-1} \\
\Rightarrow \quad \sum_{k=1}^{N} u\left(T_{k}\right)=u_{\text {sum }}>m-u_{\varphi-1} .
\end{gathered}
$$

Hence, any system that Algorithm ASSIGN fails to partition must have $u_{\text {sum }}>m-u_{\varphi-1}$.

If $\varphi$ is much smaller than $m$, which will often be the case in practice, then the proposed assignment algorithm results in little utilization loss even in the worst case.

Bounding $\varpi_{\text {sum }}$. For any given DAG system, if all DAGs can be assigned in the first phase, then $\varpi_{\text {sum }}=0$. In the second phase, each DAG is considered in order, and if a cluster fails to accommodate a task (line 15), then it will never be considered for later tasks. Thus, it immediately follows that at most $\varphi-1$ DAGs can contribute to $\varpi_{\text {sum }}$. Due to the fact that in the worst case all edges of a DAG can cause inter-cluster communication (as illustrated by the example below), an upper-bound on $\varpi_{\text {sum }}$ under Algorithm ASSIGN is given by the sum of $\varphi-1$ largest DAG data weights.

Example. Consider a scenario where three DAGs $T_{i}, T_{j}$, and $T_{k}$ are assigned to three clusters in a way as shown in Fig. 5. Note that all edges of $\varphi-1=2$ DAGs $T_{j}$ and $T_{k}$ contribute to $\varpi_{\text {sum }}$. 


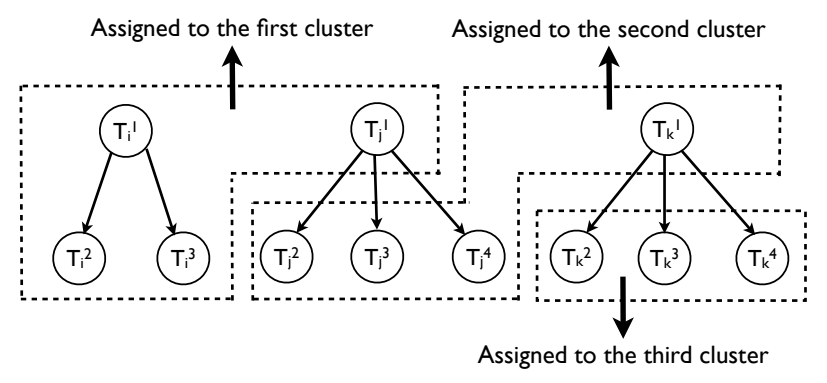

Figure 5: Example worst-case scenario where all edges of $\varphi-1$ DAGs contribute to the total communication cost.

\subsection{Scheduling Phase}

After executing the assignment phase, every task is mapped to a cluster. The scheduling phase of CDAG ensures that each task is scheduled with bounded tardiness. The scheduling phase consists of two steps: (i) transform each PGM graph into ordinary sporadic tasks by redefining job releases, and (ii) apply any window-constrained scheduling policy [13] such as GEDF to globally schedule the transformed tasks within each cluster.

Transforming PGM graphs into sporadic tasks. Our recent work has shown that on a multiprocessor, any PGM graph system can be transformed into a set of ordinary sporadic tasks without utilization loss (see [14] for details). The transformation process ensures that all precedence constraints in the original PGM graphs are met. This is done by redefining job releases properly. However, data communication delays (inter-cluster or intra-cluster) were not considered in this previous work. In this paper, for each cluster, we apply the same approach but redefine job releases in a way such that data communications are considered. Later we shall show that this process still ensures bounded tardiness for any graph.

Definition 5. Let $F_{\max }\left(\operatorname{pred}\left(T_{l, j}^{h}\right), v_{l, j}^{h}\right)$ denote the latest completion time plus the data communication time among all predecessor jobs of $T_{l, j}^{h}$, where $v_{l, j}^{h}$ denotes the time to transfer data from the corresponding predecessor job of $T_{l, j}^{h}$ to $T_{l, j}^{h}$. For any predecessor job $T_{l, i}^{k}$ of $T_{l, j}^{h}, v_{l, j}^{h}$ can be computed by dividing $\rho_{l}^{k h}$ (the number of produced data units on the corresponding edge) by the corresponding network bandwidth (i.e., $B$ for inter-cluster data communications and $b$ for intra-cluster data communications).

Definition 6. Let $t_{f}\left(T_{l, j}^{h}\right)$ denote the completion time of job $T_{l, j}^{h}$.

The following equations can be applied to redefine job releases and deadlines in an iterative way (job $T_{l, j}^{h}$ 's redefined release depends on the redefined release of $T_{l, j-1}^{h}$ where $j>1$ ).

For any job $T_{l, j}^{h}$ where $j>1$ and $h>1$, its redefined release time, denoted $r\left(T_{l, j}^{h}\right)$, is given by

$$
\begin{aligned}
r\left(T_{l, j}^{h}\right)= & \max \left(r_{l, j}^{h}, r\left(T_{l, j-1}^{h}\right)+d_{l}^{h},\right. \\
& \left.F_{\max }\left(\operatorname{pred}\left(T_{l, j}^{h}\right), v_{l, j}^{h}\right)\right) .
\end{aligned}
$$

Given that a source task has no predecessors, the redefined release of any job $T_{l, j}^{1}(j>1)$ of such a task, $r\left(T_{l, j}^{1}\right)$, is given by

$$
r\left(T_{l, j}^{1}\right)=\max \left(r_{l, j}^{1}, r\left(T_{l, j-1}^{1}\right)+d_{l}^{1}\right) .
$$

For the first job $T_{l, 1}^{h}(h>1)$ of any non-source task, its redefined release, $r\left(T_{l, 1}^{h}\right)$, is given by

$$
r\left(T_{l, 1}^{h}\right)=\max \left(r_{l, 1}^{h}, F_{\max }\left(\operatorname{pred}\left(T_{l, j}^{h}\right), v_{l, j}^{h}\right)\right) .
$$

Finally, for the first job $T_{l, 1}^{1}$ of any source task, its release time is not altered, i.e.,

$$
r\left(T_{l, 1}^{1}\right)=r_{l, 1}^{1} .
$$

(Note that when redefining job releases in our previous work, the term $v_{l, j}^{h}$ did not appear in Eqs. (5)-(7) since data communications were not considered.)

After redefining job releases according to (5)-(7), any job $T_{l, j}^{h}$ 's redefined deadline, denoted $d\left(T_{l, j}^{h}\right)$, is given by

$$
d\left(T_{l, j}^{h}\right)=r\left(T_{l, j}^{h}\right)+d_{l}^{h} .
$$

Note that these definitions imply that each task's utilization remains unchanged. In particular, as shown in Sec. 3.3, bounded tardiness can be ensured for every transformed task in any cluster. Thus, Eqs. (5)-(7) delay any job release by a bounded amount, which implies that the execution rate and the relative deadline of each task is unchanged. Note also that the release time of any job $T_{l, j}^{h}$ with predecessor jobs is redefined to be at least $F_{\max }\left(\operatorname{pred}\left(T_{l, j}^{h}\right), v_{l, j}^{h}\right)$. Hence, the schedule preserves the precedence constraints enforced by the PGM model. Furthermore, since the release time of each $T_{l, j}^{h}(j>1)$ is redefined to be at least that of $T_{l, j-1}^{h}$ plus $d_{l}^{h}, T_{l}$ executes as a sporadic task with a period of $d_{l}^{h}$.

Example. Suppose that DAG $T_{2}$ in Fig. 2 is to be assigned to clusters in a way such that $T_{1}^{1}$ and $T_{1}^{2}$ are assigned to different clusters. For any job $T_{2, j}^{2}$ of $T_{2}$, its predecessor job is $T_{2, j}^{1}$. Thus, assuming $B=2$ as in Fig. 1(c), by Def. 5, for any job $T_{2, j}^{2}$, we have $v_{2, j}^{2}=\frac{\rho_{2}^{12}}{B}=\frac{4}{2}=2$. Fig. 6(a) shows the original job releases for $T_{2}^{1}$ and $T_{2}^{2}$ and Fig. 6(b) shows the redefined job releases according to Eqs. (5)-(7) and the corresponding job executions. (Insets (c) and (d) are considered later.) Given that job $T_{2,1}^{1}$ completes at time 4, according to Eq. (7), the release of $T_{2,1}^{2}$ is redefined to be at time 6. According to Eq. (6), the release of $T_{2,2}^{1}$ is redefined to be at time 6 . Then, $T_{2,2}^{1}$ completes at time 8 . According to Eq. (5), the release of $T_{2,2}^{2}$ is redefined to be at 


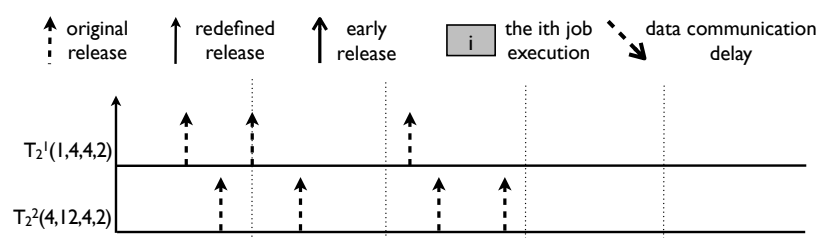

(a) Original releases

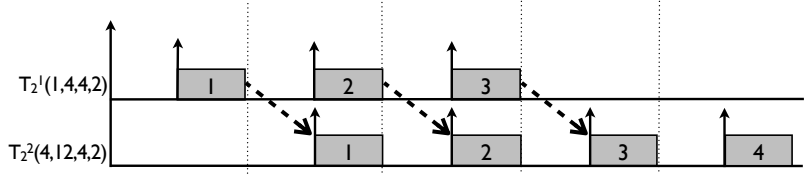

(b) Redefined releases with communication delay

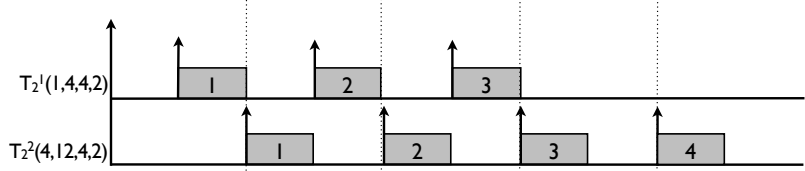

(c) Redefined releases without communication delay

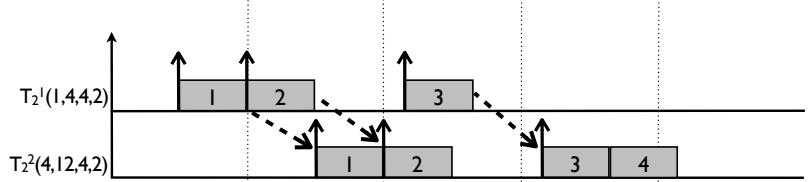

(d) Early-releasing:

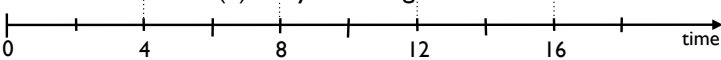

Figure 6: Illustrating various ideas on redefining job releases for DAG $T_{2}$ in Fig. 2.

time 10, which is the completion time of its predecessor job $T_{2,2}^{1}$ plus the data communication time. Similarly, releases of other jobs can be defined by Eqs. (5)-(7). Note that the redefined job releases are in accordance with the sporadic task model. Moreover, $T_{2}^{1}$ and $T_{2}^{2}$ execute as if they were ordinary sporadic tasks, and yet all precedence constraints are satisfied.

\subsection{Tardiness Bound}

Given a PGM-specified system, by applying the strategy presented above, we obtain a transformed task system $\tau$ containing only independent sporadic tasks. Then, we can use any window-constrained global scheduling algorithm [13] to schedule tasks within each cluster. It has been shown in [13] that any window-constrained global scheduling algorithm can ensure bounded tardiness for sporadic tasks on multiprocessors with no utilization loss.

In our previous work [14], we derived a tardiness bound for any PGM system scheduled on a multiprocessor (which can be considered as a single cluster, as a special case of our multi-cluster system) under GEDF, without considering the communication time, as stated in the following theorem.

Theorem 2. [14] The tardiness of any job $T_{l, j}^{h}$ of any task $T_{l}^{h}$ at depth $k$ within a DAG $T_{l}$ scheduled under GEDF on a multiprocessor is at most $(k+1) \cdot \Delta+3(k+1) \cdot y_{l}^{\max }$, where $y_{l}^{\max }=\max \left(y_{l}^{1}, y_{l}^{2}, \ldots, y_{l}^{z}\right)(z$ is the number of nodes within $T_{l}$ ) and $\Delta$ denotes the tardiness bound of $T_{l}$ with respect to its redefined deadlines, as specified in [14] (omitted here due to space constraints), i.e.,

$$
t_{f}\left(T_{l, j}^{h}\right)-d\left(T_{l, j}^{h}\right) \leq \Delta .
$$

Fig. 6(b) shows the redefined releases and the job executions after considering data communication times (as covered earlier). Fig. 6(c) shows the redefined releases and the corresponding job executions assuming no data communication time for DAG $T_{2}$. As seen, the data communication further delays the redefined releases to later points of time. By bounding such data communication times and appropriately incorporating them into the prior tardiness bound (i.e., the one assuming no communication time), we are able to derive a final tardiness bound for every task in the given PGM system scheduled under CDAG, as stated in the following theorem.

Theorem 3. The tardiness of any job $T_{l, j}^{h}$ of any task $T_{l}^{h}$ at depth $k$ within a DAG $T_{l}$ scheduled under CDAG with respect to its original deadline is at most

$$
(k+1) \cdot \Delta+3(k+1) \cdot\left(y_{l}^{\max }+\max \left(v_{l, j}^{h}\right)\right),
$$

where $\max \left(v_{l, j}^{h}\right)$ denotes the maximum data communication time between any predecessor job of $T_{l, j}^{h}$ and $T_{l, j}^{h}$.

Proof. The proof is given in an appendix.

Note that a per-task response time bound can be obtained from the above tardiness bound by adding the task's relative deadline. Such bounds are useful in settings where response time is used as the performance metric. ${ }^{4}$ Note also that since no utilization loss occurs during the scheduling phase, any PGM system is schedulable with bounded response times as long as it can be partitioned onto clusters under CDAG.

\subsection{Improving Job Response Times}

According to Eqs.(5)-(7), we delay job releases to transform DAGs into sporadic tasks. However, excessive release delays are actually unnecessary and actual response times can be improved by applying a technique called "earlyreleasing," which allows jobs to execute before their specified release times [1]. The earliest time at which job $T_{l, j}^{h}$ may execute is defined by its early-release time $\varepsilon\left(T_{l, j}^{h}\right)$, where $\varepsilon\left(T_{l, j}^{h}\right) \leq r\left(T_{l, j}^{h}\right)$. For any job $T_{l, j}^{h}$, its early-releasing time can be defined as

$$
\varepsilon\left(T_{l, j}^{h}\right)= \begin{cases}r_{l, j}^{h} & \text { if } h=1 \\ F_{\text {max }}\left(\operatorname{pred}\left(T_{l, j}^{h}\right), v_{l, j}^{h}\right) & \text { if } h>1 .\end{cases}
$$

\footnotetext{
${ }^{4}$ In some PGM-specified applications, deadlines are not specified but bounded response times are still required [10].
} 
An unfinished job $T_{l, j}^{h}$ is eligible for execution at time $t$ if $T_{l, j-1}^{h}$ has completed by $t$ (if $j>1$ ) and $t \geq \varepsilon\left(T_{l, j}^{h}\right)$. The tardiness bounds in Theorem 2 (as shown in [14]) and Theorem 3 continue to hold if early-releasing is allowed. Intuitively, this is reflective of the fact that schedulability mainly hinges on the proper spacing of consecutive job deadlines of a task, instead of its releases.

Example. Consider again the scheduling of $T_{2}$ as shown in Fig. 6(b). Fig. 6(d) shows early releases as defined above and the corresponding GEDF schedule. As seen, most jobs' response times are improved. For instance, $T_{2,2}^{2}$ now completes at time 10, two time units earlier than the case without early-releasing.

\section{Experiments}

In this section, we describe experiments conducted using randomly-generated DAG sets to evaluate the effectiveness of CDAG in minimizing utilization loss and total communication cost. We do this by comparing CDAG with the optimal ILP solution. The experiments focus on three performance metrics: (i) utilization loss, (ii) total communication cost, and (iii) each test's runtime performance.

In our experiments, we selected a random target size for DAGs, from at least one task to 100 per DAG. Then tasks within each DAG were generated based upon distributions proposed by Baker [2]. The source task of each DAG was assumed to be released sporadically, with a period uniformly distributed over $[10 \mathrm{~ms}, 100 \mathrm{~ms}]$. The produce amount of each edge was varied from 10 data units to 1000 data units. For every edge of each DAG, its produce amount, threshold, and consume amount were assumed to be the same. Valid execution rates were calculated for nonsource tasks within each DAG using results from $[10,14]$. Task utilizations were distributed using four uniform distributions, [0.05, 0.2] (light), [0.2, 0.5] (medium), [0.5, 0.8] (heavy), and $[0.05,0.8]$ (uniform). Task execution costs were calculated from execution rates and utilizations. We generated six clusters, each with a random processor count from 4 to 16, with a total processor count of 48 . We assumed $B=10$ and $b=1000$. For each choice of utilization distribution, a cap on overall utilization was systematically varied within $[16,48]$. For each combination of utilization cap and utilization distribution, we generated 100 DAG sets. Each such DAG set was generated by creating DAGs until total utilization exceeded the corresponding utilization cap, and by then reducing the last DAG's utilization so that the total utilization equalled the utilization cap.

The schedulability results that were obtained are shown in Fig. 7. In all insets of Fig. 7, "CDAG" denotes the schedulability results achieved by CDAG, "Thm. 1 Bound" denotes the worst-case utilization bound of CDAG as stated in Theorem 1, and "ILP" denotes the schedulability results achieved by ILP. Each curve in each figure plots the fraction of the generated DAG sets that the corresponding approach successfully scheduled, as a function of total utilization. (Note that the range of the $x$-axis in all insets is given by $[43,48]$.) As Fig. 7 shows, under all four utilization distributions, CDAG yields schedulability results that are very close to that achieved by ILP. Moreover, the worst-case utilization bound in Theorem 1 is reasonable. For example, under the light per-task utilization distribution, the worst-case utilization bound of CDAG ensures that any DAG set with a total utilization up to 47 can be successfully scheduled in a distributed system containing 48 processors.

Table 1 shows the total communication cost achieved by both approaches categorized by the total utilization $U_{\text {sum }}$ using the light per-task utilization distribution (we omit the results using other utilization distributions because they all show similar trends). In these experiments, all DAG sets were guaranteed to be schedulable since the total utilization of any DAG set (at most 44) is less than the worst-case utilization bound of CDAG, which is 47 . In Table 1, for each $U_{\text {sum }}$, the total communication cost under ILP or CDAG was computed by taking the average of the total communication cost over the 100 generated DAG sets. The total communication cost for each generated DAG set is given by $\varpi_{\text {sum }}$ as defined in Def. 2. The label "Total" represents the maximum communication cost of the DAG set, which is given by $\sum_{T_{i} \in \zeta} \sum_{e_{i}^{j k} \in T_{i}} w_{i}^{j k}$ where (as noted earlier) $\zeta$ represents the corresponding DAG set. As seen, CDAG is effective in minimizing the total communication cost. The total communication costs achieved by CDAG are close to the optimal ones achieved by ILP and are significantly smaller than the maximum communication costs. For example, when $U_{\text {sum }}=44$, CDAG achieves a total communication cost of 248.7 data units while ILP gives an optimal solution of 32.6 data units, both of which are almost negligible compared to the maximum communication cost, which is 93775.9 data units.

Regarding runtime performance, Table 2 shows the average time to run an experiment as a function of the number of tasks $N$ using the light per-task utilization distribution (we again omit the results using other utilization distributions because they all show similar trends). For each $N$ in the set $\{100,200,300,400,500\}$, we generated ten DAG sets and recorded the average running time of both ILP and CDAG. CDAG consistently took less than $1 \mathrm{~ms}$ to run while ILP ran for a significantly longer time, sometimes prohibitively so. For instance, when $N=500$, ILP took more than 10 hours on average per generated DAG set.

\section{Conclusion}

In this paper, we have shown that DAG-based systems with sophisticated notions of acyclic precedence constraints 


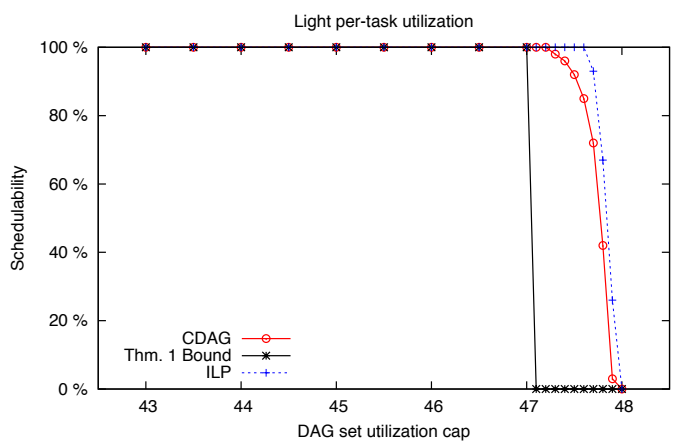

(a)

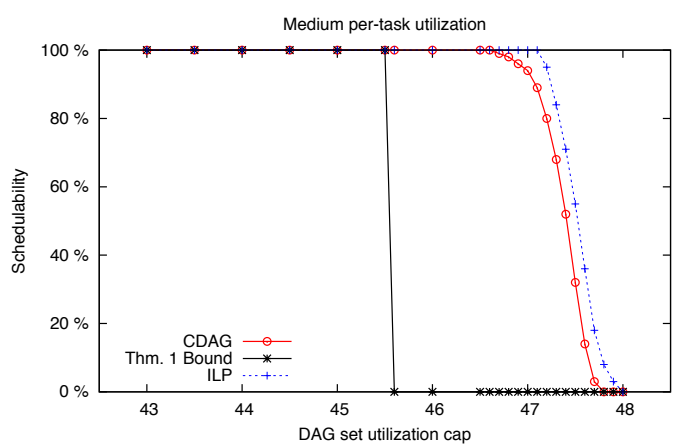

(b)

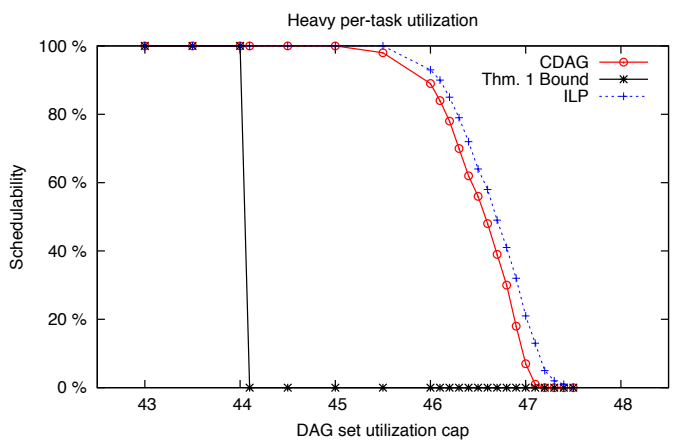

(c)

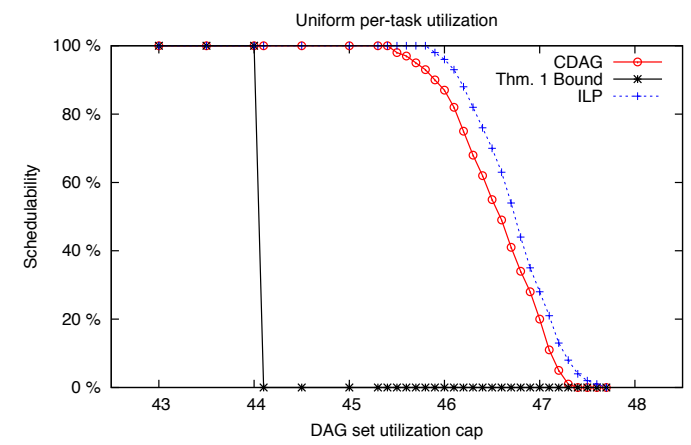

(d)

Figure 7: Schedulability results. (a) Light per-task utilization distribution. (b) Medium per-task utilization distribution. (c) Heavy per-task utilization distribution. (d) Uniform per-task utilization distribution.

can be efficiently supported under the proposed clustered scheduling algorithm CDAG in a distributed system, pro-
Table 1: Total communication cost.

\begin{tabular}{|c|c|c|c|c|c|c|c|c|}
\hline $\begin{array}{c}\text { DAG set } \\
\text { Methilization }\end{array}$ & $\mathrm{U}_{\text {sum }}=16$ & $\mathrm{U}_{\text {sum }}=20$ & $\mathrm{U}_{\text {sum }}=24$ & $\mathrm{U}_{\text {sum }}=28$ & $\mathrm{U}_{\text {sum }}=32$ & $\mathrm{U}_{\text {sum }}=36$ & $\mathrm{U}_{\text {sum }}=40$ & $\mathrm{U}_{\text {sum }}=44$ \\
\hline ILP & 6.4 & 7.5 & 10.6 & 17 & 22.5 & 26.2 & 29.8 & 32.6 \\
\hline CDAG & 78.1 & 87.2 & 130.5 & 146.3 & 173.1 & 187.7 & 231.2 & 248.7 \\
\hline Total & 34588.9 & 45048.8 & 52968.1 & 58895.3 & 69895.9 & 77125.5 & 86741.4 & 93775.9 \\
\hline
\end{tabular}

Table 2: Runtime performance.

\begin{tabular}{|c|c|c|c|c|c|}
\hline $\begin{array}{c}\text { Method } \\
\text { Mef tasks }\end{array}$ & $\mathrm{N}=100$ & $\mathrm{~N}=200$ & $\mathrm{~N}=300$ & $\mathrm{~N}=400$ & $\mathrm{~N}=500$ \\
\hline ILP & $5 I .9(\mathrm{~s})$ & $\mathrm{I} 65.9(\mathrm{~s})$ & $4 I 2.2(\mathrm{~s})$ & $2848.1(\mathrm{~s})$ & $3779 I .8(\mathrm{~s})$ \\
\hline CDAG & $0.48(\mathrm{~ms})$ & $0.58(\mathrm{~ms})$ & $0.62(\mathrm{~ms})$ & $0.56(\mathrm{~ms})$ & $0.7 \mathrm{I}(\mathrm{ms})$ \\
\hline
\end{tabular}

vided bounded deadline tardiness is acceptable. We assessed the effectiveness of CDAG in minimizing both utilization loss and total communication cost by comparing it with an optimal ILP solution. CDAG was analytically and experimentally shown to be effective in both respects and has low time complexity.

In future work, we would like to investigate more expressive resource models. For example, a distributed system may contain heterogeneous resources (such as CPUs and GPUs) and some tasks may need to access certain types of resources, which makes the task assignment problem more constrained. Moreover, it would be interesting to investigate other scheduling techniques such as semi-partitioned scheduling to achieve no utilization loss.

\section{References}

[1] J. Anderson and A. Srinivasan. Early-release fair scheduling. In Proc. of the 12th Euromicro Conf. on Real-Time Sys., pp. 35-43, 2000.

[2] T. Baker. A comparison of global and partitioned EDF schedulability tests for multiprocessors. In Technical Report TR-051101, Florida State University, 2005.

[3] T. Baker and S. Baruah. Schedulability analysis of multiprocessor sporadic task systems. In Handbook of real-time and embedded systems, Chapman Hall/CRC, Boca Raton, Florida, 2007.

[4] S. Baruah, N. Cohen, C. Plaxton, and D. Varvel. Proportionate progress: A notion of fairness in resource allocation. In Algorithmica, Vol.15, pp. 600-625, 1996.

[5] J. Calandrino, J. Anderson, and D. Baumberger. A hybrid real-time scheduling approach for large-scale multicore systems. In Proc. of the 19th ECRTS, pp. 247-256, 2007.

[6] J. Carpenter, S. Funk, P. Holman, J. Anderson, and S. Baruah. Handbook on Scheduling Algorithms, Methods, and Models. Chapman Hall/CRC, Boca, 2004. 
[7] S. Chakraborty, T. Erlebach, and L. Thiele. On the complexity of scheduling conditional real-time code. In Lecture in Computer Science, Vol. 2125, pp. 38-49, 2001.

[8] DARPA. Satellite System F6 Specification and Requirement. http://www.darpa.mil/Our_Work/TTO/Programs/Systemf6/ System_F6.aspx, 2011.

[9] M. Delest, A. Don, and J. Benois-Pineau. DAG-based visual interfaces for navigation in indexed video content. In Multimedia Tools and Applications, Vol. 31, pp. 51-72, 2006.

[10] S. Goddard. On the Management of Latency in the synthesis of real-time signal processing systems from processing graphs. $\mathrm{PhD}$ thesis, The University of North Carolina at Chapel Hill, 1998.

[11] K. Jeffay and S. Goddard. A theory of rate-based execution. In Proc. of the 20th RTSS, pp. 304-314, 1999.

[12] Naval Research Laboratory. Processing graph method specification. In prepared by the Naval Research Laboratory for use by the Navy Standard Signal Processing Program Office (PMS-412), 1987.

[13] H. Leontyev and J. Anderson. Generalized tardiness bounds for global multiprocessor scheduling. In Real-Time Systems, Vol. 44, pp. 26-71, 2010.

[14] C. Liu and J. Anderson. Supporting soft real-time DAG-based systems on multiprocessors with no utilization loss. In Proc. of the 31st RTSS, pp. 3-13, 2010.

[15] O. Sinnen. Task Scheduling for Parallel Systems. Wiley, 2007.

\section{Appendix A}

\section{Proof for Theorem 3.}

Before proving Theorem 3, we first state two lemmas that have been proved in [14] for systems without considering data communications.

Lemma 2. For any job $T_{l, j}^{1}, r\left(T_{l, j}^{1}\right)-r_{l, j}^{1}<2 \cdot y_{l}^{1}$.

Proof. By (6), $r\left(T_{l, j}^{1}\right)$ is lndependent of the data communication time. Thus, the proof is exactly the same as the one for proving Lemma 3 in [14].

Lemma 3. For any two jobs $T_{l, j}^{h}$ and $T_{l, k}^{h}$ of $T_{l}^{h}$, where $j<$ $k, i \cdot y_{l}^{h} \leq r_{l, j}^{h}<(i+1) \cdot y_{l}^{h}(i \geq 0)$, and $(i+w) \cdot y_{l}^{h} \leq$ $r_{l, k}^{h}<(i+w+1) \cdot y_{l}^{h}(w \geq 0)$, we have $r_{l, k}^{h}-r_{l, j}^{h}>$ $(k-j) \cdot d_{l}^{h}-2 \cdot y_{l}^{h}$.

Proof. The objective of this lemma is to prove the stated properties on $r_{l, k}^{h}$ and $r_{l, j}^{h}$, which are the original releases of any two jobs $T_{l, j}^{h}$ and $T_{l, k}^{h}$ of any task $T_{l}^{h}$. Thus, the proof does not involve any data communication time. Therefore, the proof is exactly the same as the one for proving Lemma 4 in [14].
Now we prove Theorem 3.

Proof. This theorem can be proved by induction on task depth. In the base case, by Theorem 2 and the fact that $T_{l}^{1}$ has no predecessors, its tardiness with respect to its newly-defined deadline, $d\left(T_{l, j}^{1}\right)$, is at most $\Delta$. By Lemma 2, $r\left(T_{l, j}^{1}\right)-r T_{l, j}^{1}<2 \cdot y_{l}^{1}$. Thus, with respect to its original deadline, $d T_{l, j}^{1}, T_{l, j}^{1}$ has a tardiness bound of $\Delta+2 \cdot y_{l}^{1}<$ $\Delta+3 \cdot y_{l}^{\max }$.

For the induction step, let us assume (11) holds for any task $T_{l}^{w}$ at depth at most $k-1, k \geq 1$. Then, the tardiness of any job $T_{l, v}^{w}$ of $T_{l}^{w}$ is at most $k \cdot \Delta+3 k \cdot\left(y_{l}^{\max }+\max \left(v_{l, j}^{h}\right)\right)$, i.e.,

$$
t_{f}\left(T_{l, v}^{w}\right)-d_{l, v}^{w} \leq k \cdot \Delta+3 k \cdot\left(y_{l}^{\max }+\max \left(v_{l, j}^{h}\right)\right) .
$$

We want to prove that for any job $T_{l, j}^{h}$ of any task $T_{l}^{h}$ at depth $k, t_{f}\left(T_{l, j}^{h}\right)-d_{l, j}^{h} \leq(k+1) \cdot \Delta+3(k+1) \cdot\left(y_{l}^{\max }+\max \left(v_{l, j}^{h}\right)\right)$. According to (5) and (7), there are three cases to consider regarding $T_{l, j}^{h}$ 's newly-defined release time $r\left(T_{l, j}^{h}\right)$.

Case 1. $r\left(T_{l, j}^{h}\right)=r_{l, j}^{h}$. By Theorem 2, we know that $t_{f}\left(T_{l, j}^{h}\right)-d\left(T_{l, j}^{h}\right) \leq \Delta$. Given that $d\left(T_{l, j}^{h}\right)=d_{l, j}^{h}$, we have $t_{f}\left(T_{l, j}^{h}\right)-d_{l, j}^{h} \leq \Delta<(k+1) \cdot \Delta+3(k+1) \cdot\left(y_{l}^{\max }+\right.$ $\left.\max \left(v_{l, j}^{h}\right)\right)$.

Case 2. $r\left(T_{l, j}^{h}\right)=F_{\max }\left(\operatorname{pred}\left(T_{l, j}^{h}\right), v_{l, j}^{h}\right)$. Let $T_{l, v}^{w}$ be the predecessor of $T_{l, j}^{h}$ that has the latest completion time among all predecessors of $T_{l, j}^{h}\left(T_{l, v}^{w}\right.$ exists because the depth of $T_{l}^{h}$ is at least one). Thus, we have

$$
r\left(T_{l, j}^{h}\right)=F_{\max }\left(\operatorname{pred}\left(T_{l, j}^{h}\right), v_{l, j}^{h}\right) \leq t_{f}\left(T_{l, v}^{w}\right)+\max \left(v_{l, j}^{h}\right) .
$$

Therefore,

$$
\begin{aligned}
& t_{f}\left(T_{l, j}^{h}\right)-d_{l, j}^{h} \\
& \{\text { by }(2)\} \\
& =t_{f}\left(T_{l, j}^{h}\right)-r_{l, j}^{h}-d_{l}^{h} \\
& =t_{f}\left(T_{l, j}^{h}\right)-r\left(T_{l, j}^{h}\right)+r\left(T_{l, j}^{h}\right)-r_{l, j}^{h}-d_{l}^{h} \\
& \{\text { by }(9)\} \\
& =t_{f}\left(T_{l, j}^{h}\right)-d\left(T_{l, j}^{h}\right)+d_{l}^{h}+r\left(T_{l, j}^{h}\right)-r_{l, j}^{h}-d_{l}^{h} \\
& \{\text { by }(10)\} \\
& \leq \Delta+d_{l}^{h}+r\left(T_{l, j}^{h}\right)-r_{l, j}^{h}-d_{l}^{h} \\
& =\Delta+r\left(T_{l, j}^{h}\right)-r_{l, j}^{h} \\
& \{\text { by (3) and (13) } \\
& \leq \Delta+t_{f}\left(T_{l, v}^{w}\right)+\max \left(v_{l, j}^{h}\right)-r_{l, v}^{w} \\
& \{\text { by (2)\} } \\
& =\Delta+t_{f}\left(T_{l, v}^{w}\right)+\max \left(v_{l, j}^{h}\right)-d_{l, v}^{w}+d_{l}^{w} \\
& \{\text { by }(12)\} \\
& \leq \Delta+k \cdot \Delta+3 k \cdot\left(y_{l}^{\max }+\max \left(v_{l, j}^{h}\right)\right) \\
& +\max \left(v_{l, j}^{h}\right)+d_{l}^{w} \\
& \{\text { by }(1)\} \\
& <(k+1) \cdot \Delta+3(k+1) \cdot\left(y_{l}^{\max }+\max \left(v_{l, j}^{h}\right)\right) \text {. }
\end{aligned}
$$


Case 3. $j>1 \wedge r\left(T_{l, j}^{h}\right)=r\left(T_{l, j-1}^{h}\right)+d_{l}^{h}$. Let $T_{l, q}^{h}$ $(q<j)$ denote the last job of $T_{l}^{h}$ released before $T_{l, j}^{h}$ such that $r\left(T_{l, q}^{h}\right)=r_{l, q}^{h}$ or $r\left(T_{l, q}^{h}\right)=F_{\max }\left(\operatorname{pred}\left(T_{l, q}^{h}\right), v_{l, j}^{h}\right)$. $T_{l, q}^{h}$ exists because according to (7) and (8), there exists at least one job, $T_{l, 1}^{h}$, such that $r\left(T_{l, 1}^{h}\right)=r_{l, 1}^{h}$ or $r\left(T_{l, 1}^{h}\right)=$ $F_{\text {max }}\left(\operatorname{pred}\left(T_{l, 1}^{h}\right), v_{l, j}^{h}\right)$. Depending on the value of $r\left(T_{l, q}^{h}\right)$, we have two subcases.

Case 3.1. $r\left(T_{l, q}^{h}\right)=r_{l, q}^{h}$. By the definition of $T_{l, q}^{h}$, the release time of any job $T_{l, k}^{h}$, where $q<k \leq j$, is redefined to be $r\left(T_{l, k}^{h}\right)=r\left(T_{l, k-1}^{h}\right)+d_{l}^{h}$. Thus, we have

$$
r\left(T_{l, j}^{h}\right)=r\left(T_{l, q}^{h}\right)+(j-q) \cdot d_{l}^{h} .
$$

Therefore, we have

$$
\begin{aligned}
& t_{f}\left(T_{l, j}^{h}\right)-d_{l, j}^{h} \\
& \{\text { by }(2)\} \\
= & t_{f}\left(T_{l, j}^{h}\right)-r_{l, j}^{h}-d_{l}^{h} \\
= & t_{f}\left(T_{l, j}^{h}\right)-r\left(T_{l, j}^{h}\right)+r\left(T_{l, j}^{h}\right)-r_{l, j}^{h}-d_{l}^{h} \\
& \{\text { by }(9)\} \\
= & t_{f}\left(T_{l, j}^{h}\right)-d\left(T_{l, j}^{h}\right)+d_{l}^{h}+r\left(T_{l, j}^{h}\right)-r_{l, j}^{h}-d_{l}^{h} \\
& \{\text { by }(10)\} \\
\leq & \Delta+d_{l}^{h}+r\left(T_{l, j}^{h}\right)-r_{l, j}^{h}-d_{l}^{h} \\
= & \Delta+r\left(T_{l, j}^{h}\right)-r_{l, j}^{h} \\
& \{\text { by }(14) \text { and Lemma } 3\} \\
< & \Delta+\left(r\left(T_{l, q}^{h}\right)+(j-q) \cdot d_{l}^{h}\right)-\left(r_{l, q}^{h}\right. \\
& \left.+(j-q) \cdot d_{l}^{h}-2 \cdot y_{l}^{h}\right) \\
& \{\text { by the case condition }\} \\
= & \Delta+2 \cdot y_{l}^{h} \\
< & (k+1) \cdot \Delta+3(k+1) \cdot\left(y_{l}^{\max }+\max \left(v_{l, j}^{h}\right)\right) .
\end{aligned}
$$

Case 3.2. $\quad r\left(T_{l, q}^{h}\right)=F_{\text {max }}\left(\operatorname{pred}\left(T_{l, q}^{h}\right), v_{l, j}^{h}\right)$. Let $T_{l, v}^{w}$ denote a predecessor job of $T_{l, q}^{h}$ with $t_{f}\left(T_{l, v}^{w}\right)=$ $F_{\text {max }}\left(\operatorname{pred}\left(T_{l, q}^{h}\right), v_{l, j}^{h}\right)-d c_{l, q}^{h}\left(T_{l, v}^{w}\right)=r\left(T_{l, q}^{h}\right)-d c_{l, q}^{h}\left(T_{l, v}^{w}\right)$, where $d c_{l, q}^{h}\left(T_{l, v}^{w}\right)$ denotes the data communication time between $T_{l, v}^{w}$ and $T_{l, q}^{h}$. We have

$$
\begin{aligned}
& t_{f}\left(T_{l, j}^{h}\right)-d_{l, j}^{h} \\
&\{\text { similarly to the derivation in Case 3.1 }\} \\
&< \Delta+\left(r\left(T_{l, q}^{h}\right)+(j-q) \cdot d_{l}^{h}\right)-\left(r_{l, q}^{h}\right. \\
&\left.+(j-q) \cdot d_{l}^{h}-2 \cdot y_{l}^{h}\right) \\
&= \Delta+r\left(T_{l, q}^{h}\right)-r_{l, q}^{h}+2 \cdot y_{l}^{h} \\
&\{\text { by the case condition and }(3)\} \\
& \leq \Delta+t_{f}\left(T_{l, v}^{w}\right)+d c_{l, q}^{h}\left(T_{l, v}^{w}\right)-r_{l, v}^{w}+2 \cdot y_{l}^{h} \\
&\{\text { by }(2)\} d_{l}^{w} \\
&= \Delta+t_{f}\left(T_{l, v}^{w}\right)+d c_{l, q}^{h}\left(T_{l, v}^{w}\right)-d_{l, v}^{w}+d_{l}^{w}+2 \cdot y_{l}^{h} \\
& \leq\{\text { by }(12)\} \\
& \Delta+k \cdot \Delta+3 k \cdot\left(y_{l}^{\max }+\max \left(v_{l, j}^{h}\right)\right)+d c_{l, q}^{h}\left(T_{l, v}^{w}\right) \\
&+d_{l}^{w}+2 \cdot y_{l}^{h} \\
&\{\text { by }(1)\} \\
&<(k+1) \cdot \Delta+3(k+1) \cdot\left(y_{l}^{\max }+\max \left(v_{l, j}^{h}\right)\right) .
\end{aligned}
$$

